\title{
Variation improvement of mechanical properties of Mg-9Al-1Zn alloy with melt conditioned high pressure die casting
}

\author{
Y. Zhang*, J.B. Patel, Y. Wang and Z. Fan
}

Brunel Centre for Advanced Solidification Technology (BCAST), Brunel University London, Kingston Lane, Uxbridge UB8 3PH, United Kingdom

*Corresponding author. Tel.: +441895268538; Fax: +441895269758; E-mail address: yijie.zhang@brunel.ac.uk.

\begin{abstract}
Melt conditioning treatment via high shearing on $\mathrm{Mg}-9 \mathrm{Al}-1 \mathrm{Zn}$ melt was employed to disperse the oxide inclusions, and to harness the well-dispersed particles as the effective heterogeneous nucleation sites for primary $\alpha-\mathrm{Mg}$ grains during solidification in high pressure die casting. To reveal the mechanism of property improvement, Prefil pressure filtration cleanliness analyser, field-emission scanning electron microscopy with energy dispersive X-ray spectroscopy and high-resolution transmission electron microscopy were employed in present study. The experimental results showed that the application of high shearing not only improved elongation, ultimate tensile strength and yield strength simultaneously, but also reduced the variation of the mechanical properties of Mg-9Al-1Zn alloy. The improvement was ascribed to the refinement of the primary $\alpha-\mathrm{Mg}$ grains formed in both shot sleeve and die cavity, and to the reduction of porosity as well as refinement of $\mathrm{Mg}_{17} \mathrm{Al}_{12}$ phases.
\end{abstract}

Keywords: Grain refining; Variation of Mechanical Properties; Die casting; Magnesium alloys 


\section{Introduction}

Mg-9Al-1Zn (AZ91D) components produced by high pressure die casting (HPDC) became more attractive in automotive field to achieve the light weighting of vehicles due to the reasonable comprehensive mechanical properties of alloy and its good castability [1,2]. Besides the advantage of HPDC of high speed production, small thickness, good surface finish and closer dimensional tolerance [3], the main defects of this technology are the formation of porosity and defect band and then result in the deterioration of mechanical properties and the enlargement of the properties variation [3-5]. More importantly, the presence of such kind of defects is inevitable in term of the characteristics of HPDC processing. During the HPDC process, the melt was poured into shot sleeve, and about 1-2 s later the melt was pushed into die cavity by plunge with given filling speed within a short time of about $3 \mathrm{~ms}$. In view of the special process of HPDC, there are two steps of solidification. The first solidification occurs inside shot sleeve and about $20 \%$ solid phases were formed in this stage [6]. The second step is that this kind of melt was forced into the die cavity and solidified under a high pressure of about 50-100 MPa. With fast filling and cooling process, the existence of porosity in final product was inevitable. Therefore, the product was only used at as-cast state and cannot be suffered solution heat treatment for further improving mechanical strength as normal casting method did. This kind of porosity was mainly caused by entrapped air and the formation of defect band consisted of segregation and/or shrinkage porosity followed the contour parallel to the surface of the casting. Furthermore, the formation of $\mathrm{MgO}$ oxide films is also inevitable during the melt process of $\mathrm{Mg}$. And such kind of film cannot supply the protection for melt to avoid further oxidation due to its porous 
structure and volume shrinkage of about $27 \%$ [7]. Therefore, the growth of $\mathrm{MgO}$ film on surface of molten $\mathrm{Mg}$ would continuously develop without proper protection. Therefore, the existence of the oxide film increases the possibility of entrapment into final products, and forms defects and subsequently deteriorates the properties of product. Hence, the first solidified primary $\mathrm{Mg}$, porosity, inclusions and the defect band are the main factors determined the variation of mechanical properties of products.

In the past decades, many efforts from both filling process and solidification side were taken to minimize the properties variation. Optimization of the filling parameters, such as increasing the temperature of shot sleeve and pouring temperature [4], would benefit the quality of defect band by decreasing the quantity of coarse primary $\alpha-\mathrm{Mg}$ formed in shot sleeve. But it cannot improve the solidification process fundamentally and reduce the variation of mechanical properties. Grain refinement is the best way to reduce the defects by improving the filling and solidification process and to enhance the strength and elongation of materials simultaneously. For aluminium industry, there already has commercial A1TiB refiner used for refining most kinds of $\mathrm{Al}$ alloys [8-13], and grain refining is a necessary and standard procedure before casting to improve the quality of component. Magnesium alloys can be generally classified into two groups of Al free alloys and $\mathrm{Al}$ bearing alloys [14]. The Al free alloys can be refined by $\operatorname{Zr}$ [15], and Al bearing Mg alloys can be refined by master alloy containing $\mathrm{Ca}$ [16,17], $\mathrm{Sr}$ [18], B [19], $\mathrm{Al}_{4} \mathrm{C}_{3}$ [20-24], $\mathrm{TiC}$ [25], $\mathrm{SiC}[15], \mathrm{TiB}_{2}$ [26,27]. The ideal refining mechanisms and commercial grain refiner are still developing. Although, good refinement results can be obtained by this kind of refinement method, it cannot remove the oxide films thoroughly and eliminate the adverse influence of oxide films. 
Recently, high shear melt conditioning technology (HSMC) developed by BCAST had been proved that it can refine all kinds of $\mathrm{Mg}$ alloys efficiently by obtaining sufficient heterogeneous nuclei particles from the dispersion of existing oxide films, in which high shearing rate was employed to break down the oxide films to nano particles [28-30]. The semi-coherent interface between $\mathrm{MgO}$ and $\mathrm{Mg}$ with a misfit of $5.46 \%$ was confirmed and the nucleation potency of $\mathrm{MgO}$ particles was testified by Fan et al. [28], Hua et al. [31] and Yun et al. [32].

In present paper, to study the variation of mechanical properties of AZ91D alloy with HPDC processing, HSMC prior to HPDC (MC-HPDC) performance was employed to produce the tensile samples for mechanical properties variation investigation. The mechanisms of the effect of high shear on solidification in both shot sleeve and die cavity were discussed.

\section{Experimental}

A commercial AZ91D Mg alloy (8.6 wt\% Al, $0.75 \mathrm{wt} \% \mathrm{Zn}, 0.25 \mathrm{wt} \% \mathrm{Mn}$ ) was employed in present study. The AZ91D alloy of $12 \mathrm{Kg}$ was melted in a Fe crucible at $650{ }^{\circ} \mathrm{C}$ under a protective atmosphere of $\mathrm{N}_{2}$ containing $0.5 \% \mathrm{SF}_{6}$. The melt conditioning treatment was conducted with a rotor-stator high shear unit within the crucible at $650^{\circ} \mathrm{C}$ under the same protective atmosphere of mixed $\mathrm{N}_{2}$ and $\mathrm{SF}_{6}$ and a controlled speed and time [28,31,33]. To obtain the optimised high shearing parameters for HPDC application, the shearing time up to $30 \mathrm{mins}$ and shearing speed at $1500 \mathrm{rpm}$ were employed in present study. TP-1 method was used herein to assess the grain size of AZ91D alloy with and without melt conditioning [34]. After shearing, the melt was 
pouring into shot sleeve for HPDC performance shot by shot. Three round samples with diameter of $6.35 \mathrm{~mm}$ were obtained for each shot. For comparison, HPDC of AZ91D alloy without shearing was obtained with the same parameter of MC-HPDC used.

The tensile test was conducted on the Instron 5500 machine with ramp rate of 1 $\mathrm{mm} / \mathrm{min}$ at room temperature. Samples for grain size and microstructure observation were cut from the middle section of testing bars, prepared by a standard procedure and then etched with mixed solution $\left(50 \mathrm{ml} / \mathrm{H}_{2} \mathrm{O}, 150 \mathrm{ml} /\right.$ ethanol and $1 \mathrm{ml} /$ acetic acid) for 2 mins. To observe the morphology of oxide film with and without high shearing, a prefil pressure filtration cleanliess analyzer (ABB Inc., Germany) was employed to concentrate and collect the oxide films and particles. Field-emission scanning electron microscopy (FE-SEM, SUPRA 35VP, Carl-Zeiss Company) with an energy dispersive X-ray spectroscopy (EDS) was used for microstructure analysis. High-resolution transmission electron microscopy (HR-TEM, JEOL 2100F, JEOL Ltd. Tokyo, Japan) operating at $200 \mathrm{kV}$ was employed for the particle/matrix interface observation.

\section{Results and Discussion}

Fig 1 shows the effect of shearing time on the grain refinement performance of AZ91D alloy. It can be seen that the grain size is decreasing with increasing of shearing time up to 25 mins. The grain size of prolonged shearing time of $30 \mathrm{mins}$ is kept at the same level of that with 25 mins shearing time. It indicates that the best shearing time for present experiment is about $30 \mathrm{mins}$. Therefore, the melt conditioning treatment for MC-HPDC in present study was conducted with the shearing parameters of 30mins shearing time, $1500 \mathrm{rpm}$ shearing speed and the melt temperature of $650^{\circ} \mathrm{C}$. 
Fig. 1 Grain refinement assessed by TP-1 method showing the trend of grain size of AZ91D alloy with various shearing time.

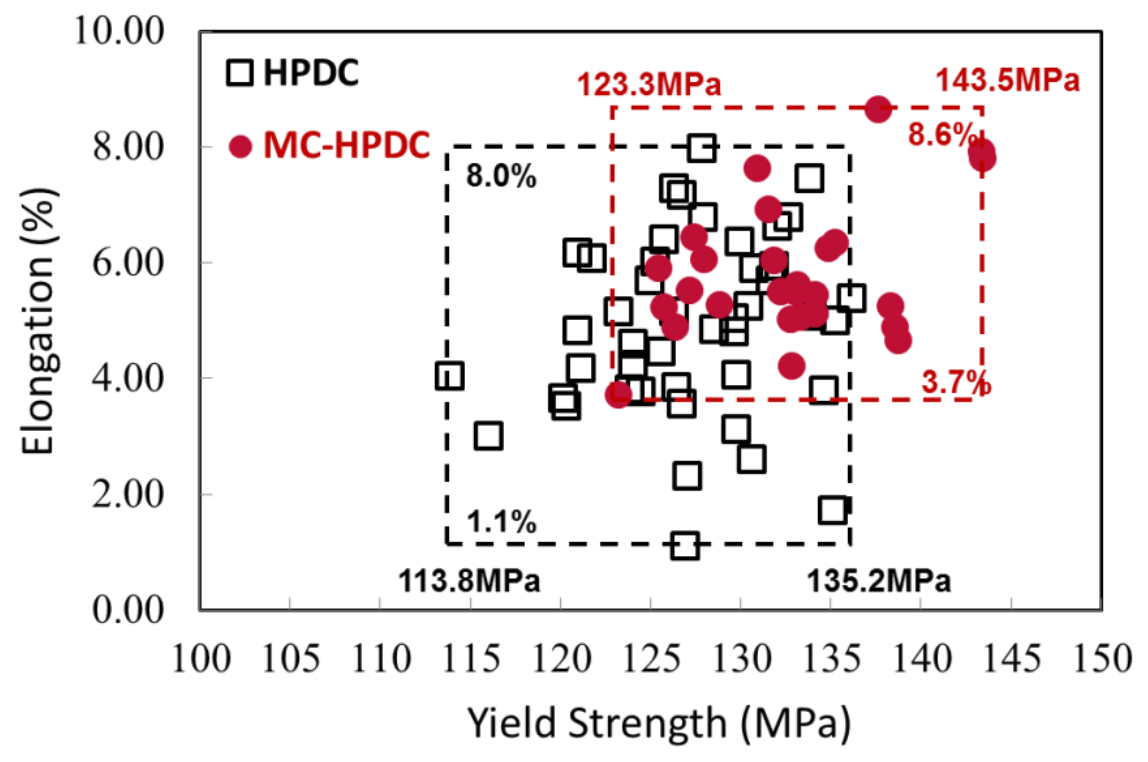

Fig. 2 Tensile test of HPDC samples showing the distribution of elongation and yield strength of AZ91D alloys with and without high shearing application.

Fig. 2 shows the comparison of tensile properties distribution of HPDC AZ91D alloy with and without high shearing application. The data in Fig. 2 indicates that the range of 
yield strength (YS) distribution of AZ91D with traditional HPDC is from $113.8 \mathrm{MPa}$ to 135.2 $\mathrm{MPa}$, and the elongation $(\delta)$ is from $1.1 \%$ to $8.0 \%$. With MC-HPDC, the yield strength distribution is from $123.3 \mathrm{MPa}$ to $143.5 \mathrm{MPa}$, the elongation is from $3.7 \%$ to 8.6\%. From the comparison, it can be seen that both the yield strength and the elongation were enhanced with application of high shearing, and the lowest yield strength and elongation are increasing by $9.5 \mathrm{MPa}$ and $2.6 \%$ respectively. This kind of improvement will be meaningful for real products structure design and material selection. For the average value of properties, the yield strength increases from 127.1 $\mathrm{MPa}$ to $132.7 \mathrm{MPa}$, and the elongation is from $4.91 \%$ to $5.79 \%$, as shown in Table 1 .

Table 1 The average value, standard deviation and coefficient of variation of yield strength and elongation of AZ91D alloy with HPDC and MC-HPDC.

\begin{tabular}{|c|c|c|c|c|c|c|}
\hline & \multicolumn{2}{|c|}{ Average $(\bar{x})$} & \multicolumn{2}{|c|}{$\begin{array}{c}\text { Standard } \\
\text { Deviation* }\end{array}$} & \multicolumn{2}{|c|}{ Coefficient of Variation } \\
\hline & $\begin{array}{c}\text { YS } \\
\text { (MPa) }\end{array}$ & $\begin{array}{c}\delta \\
(\%)\end{array}$ & $\begin{array}{c}\text { YS } \\
\text { (MPa) }\end{array}$ & $\begin{array}{c}\delta \\
(\%)\end{array}$ & $\begin{array}{l}\text { YS } \\
(\%)\end{array}$ & $\begin{array}{c}\delta \\
(\%)\end{array}$ \\
\hline HPDC & 127.1 & 4.91 & 5.02 & 1.53 & 3.95 & 31.2 \\
\hline MC-HPDC & 132.7 & 5.79 & 5.10 & 1.14 & 3.84 & 19.7 \\
\hline
\end{tabular}

Basing on the standard deviation (SD) and average value (AV), the coefficient of variation $(\mathrm{CV})$ is calculated according the equation of $\mathrm{CV}=\mathrm{SD} / \mathrm{AV}$ and the results are shown in table 1. With MC-HPDC, the variation of yield strength is decreased from $3.95 \%$ to $3.84 \%$, and the elongation is from $31.2 \%$ down to $19.7 \%$. Compared to the yield strength, the elongation has a significant improvement on coefficient of variation. The factors mainly influenced yield strength are the grain size, the size and distribution of the second phases and precipitates. For elongation, besides the grain size, the critical 
factors are the porosity and inclusions. Therefore, the improvement of mechanical properties and coefficient of variation indicates that the application of high shear melt conditioning will enhance the melt quality and subsequently determine the solidification process and microstructure evolution during HPDC performance.
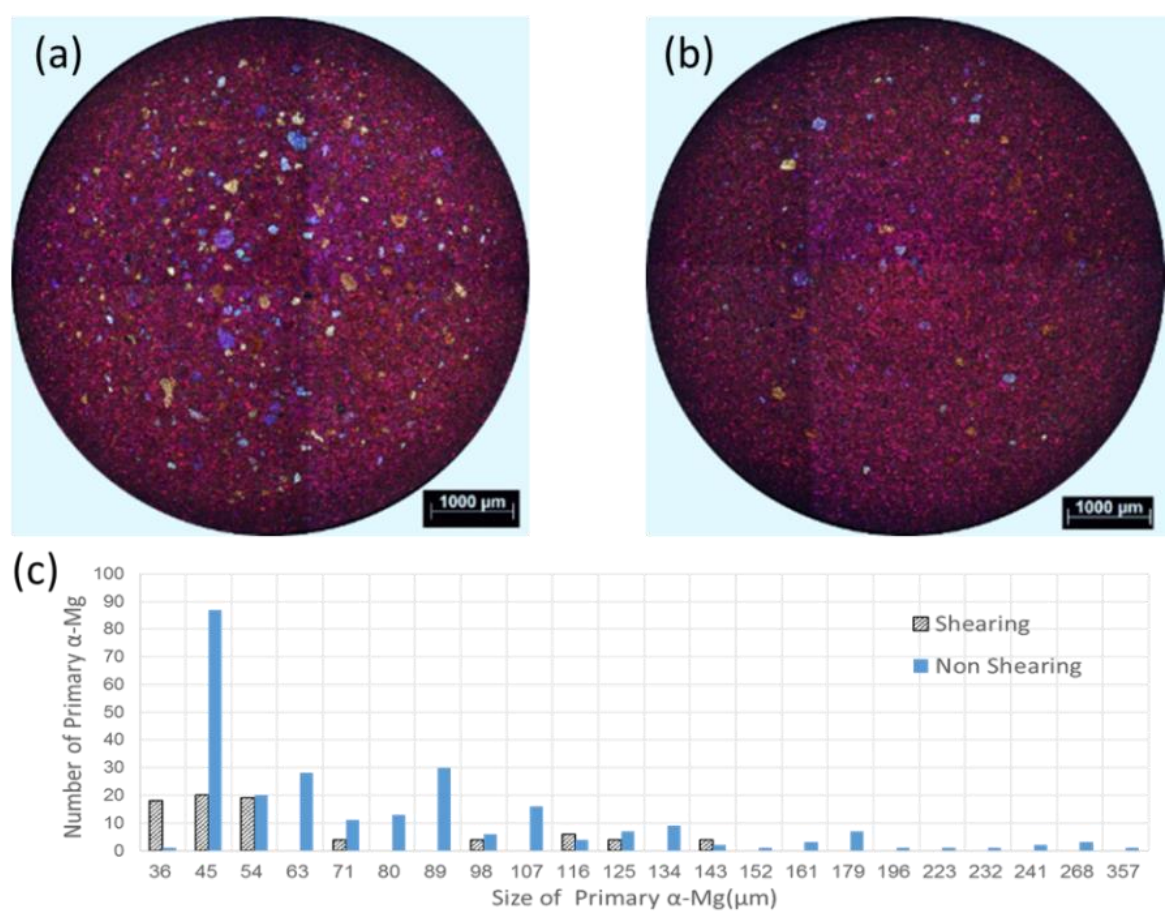

Figure 3 Microstructure of AZ91D HPDC tensile samples showing the coarse primary $\alpha-\mathrm{Mg}$ grains formed in shot sleeve (a) with and (b) without high shear melt conditioning, and (c) the grain size distribution of coarse primary $\alpha-\mathrm{Mg}$ formed in shot sleeve with and without high shearing.

The micro grain size of AZ91D tensile samples with HPDC and MC-HPDC is shown in figure 3. It can be seen from Fig.3a that a number of coarse primary $\alpha$-Mg grains was observed with traditional HPDC process. The size distribution of coarse primary $\alpha-\mathrm{Mg}$ grains is range from $30 \mu \mathrm{m}$ to $360 \mu \mathrm{m}$ and mainly between $45 \mu \mathrm{m}$ and $110 \mu \mathrm{m}$, as shown in Fig.3c. After applying high shear, both the size and quantity of primary $\alpha-\mathrm{Mg}$ 
grains were reduced, as shown in Fig. $3 b$ and c. The main size distribution range is between $30 \mu \mathrm{m}$ and $55 \mu \mathrm{m}$. The coarse primary $\alpha-\mathrm{Mg}$ observed in figure 3 is formed during the first solidification inside shot sleeve. In this stage, the solid fraction of melt is about $20 \%$, and the feature of solid part would retain at the final products and affect the formation of defect band and shrinkage porosity caused by feeding process. Therefore, the grain size of primary $\alpha-\mathrm{Mg}$ formed in shot sleeve plays an important role on the mechanical properties of final product.

In view of the staying time of AZ91D melt inside shot sleeve less than $3 \mathrm{~s}$, it means that only the melt in the outer surface contacted with shot sleeve mould has the opportunity to solidify. Therefore, it is very important to reveal the grain size of this area due to the characteristic of grains will retain as a part of microstructure of final component and affect the mechanical properties. To study the grain size of primary $\alpha-\mathrm{Mg}$ during the shot sleeve solidification, the AZ91D melt was pouring into shot sleeve as normal procedure and then leave it solidified inside shot sleeve without further performance of HPDC process. The micro grain size at the bottom of the shot sleeve solidified samples of AZ91D without and with high shearing is shown in Fig.4. It indicates that the average grain size is about $500 \pm 150 \mu \mathrm{m}, 200 \pm 50 \mu \mathrm{m}$ for samples without and with application of high shearing respectively. The refinement results testify that with melt conditioning, the nucleation at the first solidification inside shot sleeve was enhanced by high shearing. This result is well agreed with the previous report of the effect of high shearing on grain refinement of AZ91D and Mg alloy [28,29,31,33,35]. 

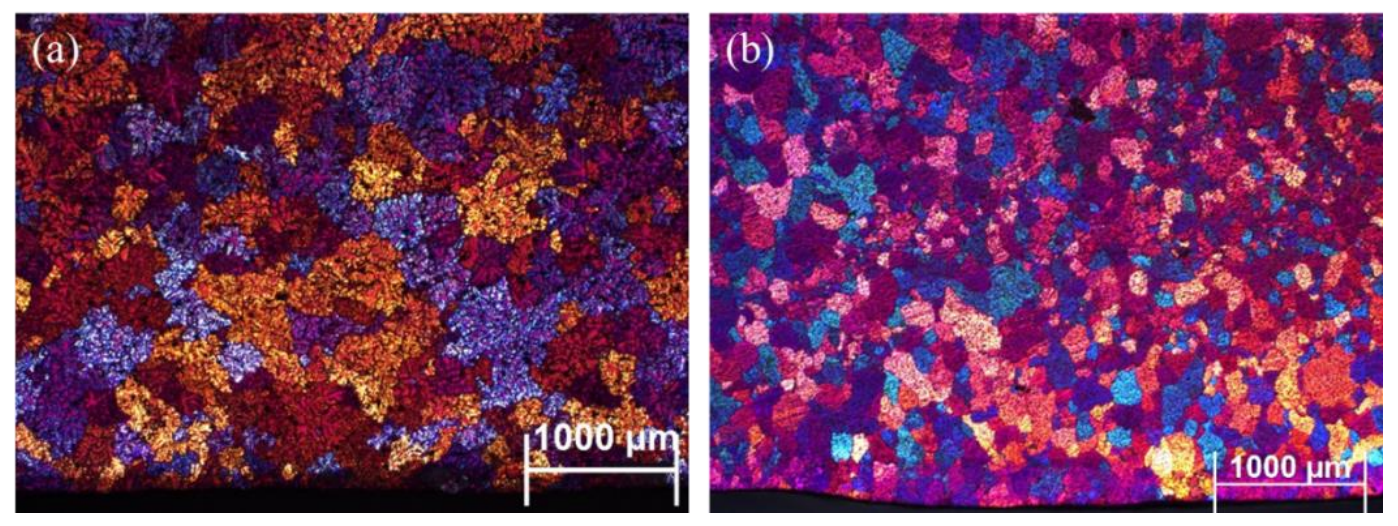

Figure 4 Microstructures showing the grain size of AZ91D ingot solidified inside shot sleeve (a) without and (b) with high shearing application.
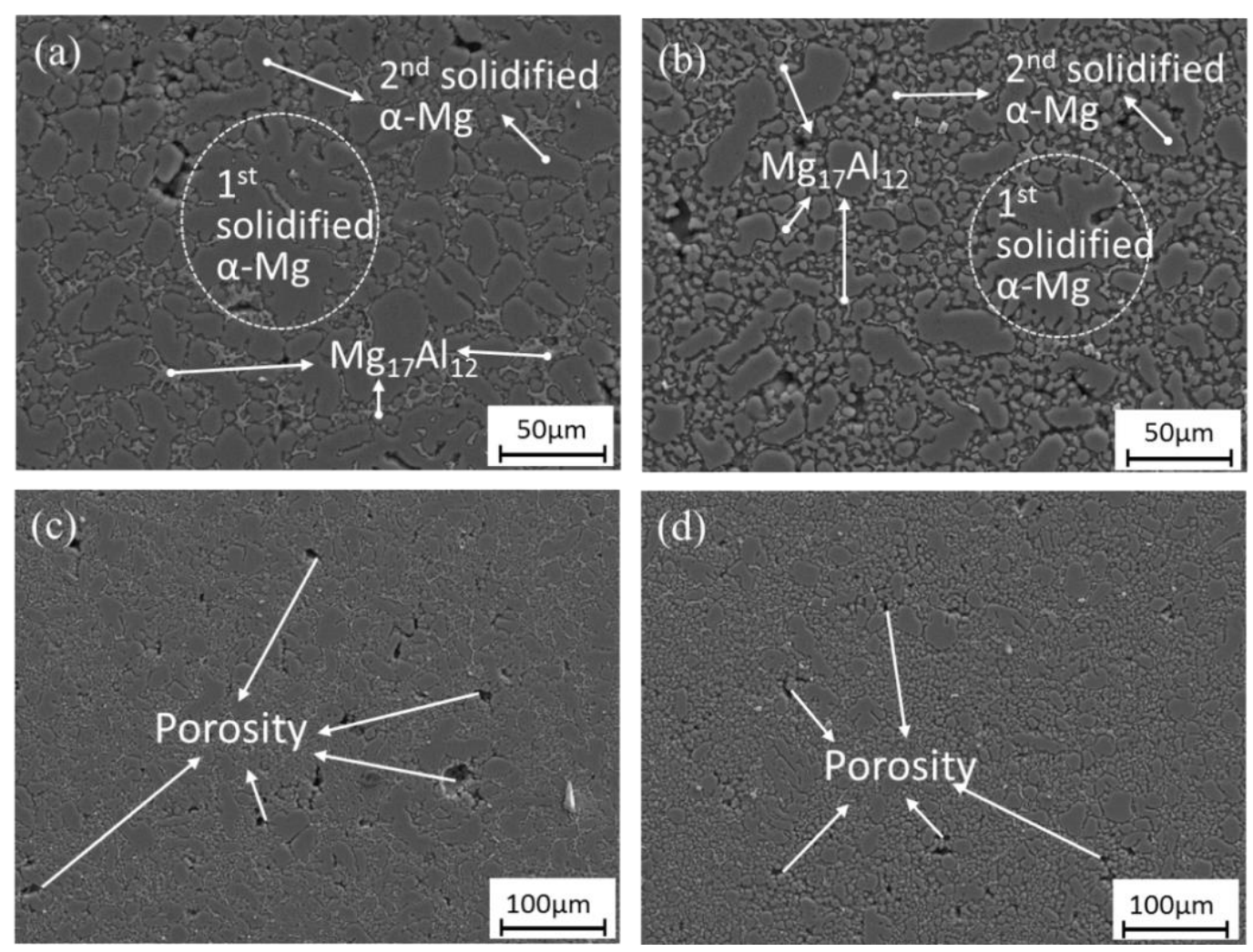

Figure 5 SEM micrographs showing the microstructure of $\alpha$-Mg grains solidified inside the shot sleeve ( $1^{\text {st }}$ solidified $\left.\alpha-M g\right)$ and inside the die cavity ( $2^{\text {nd }}$ solidified $\left.\alpha-M g\right)$, $\mathrm{Mg}_{17} \mathrm{Al}_{12}$ eutectic phase and the porosity distribution of the AZ91D alloy (a, c) without and $(b, d)$ with high shearing being imposed. 
Besides the refinement of coarse primary $\alpha-\mathrm{Mg}$ grains formed inside shot sleeve $\left(1^{\text {st }}\right.$ solidified $\alpha-\mathrm{Mg}$ ), the primary $\alpha-\mathrm{Mg}$ phases formed within the die cavity ( $2^{\text {nd }}$ solidified $\alpha-\mathrm{Mg}$ ) were also refined by applying high shearing. Figure $5 \mathrm{a}$ and $5 \mathrm{~b}$ show the size and morphology of the $1^{\text {st }}$ solidified $\alpha-\mathrm{Mg}$ and $2^{\text {nd }}$ solidified $\alpha-\mathrm{Mg}$ with HPDC and MC-HPDC respectively. The average grain size of the $2^{\text {nd }}$ solidified $\alpha-\mathrm{Mg}$ is about $20 \pm$ $5 \mu \mathrm{m}$ for AZ91D with HPDC. When high shearing is imposed, the size of the $2^{\text {nd }}$ solidified $\alpha-\mathrm{Mg}$ grains is reduced to $5-10 \mu \mathrm{m}$. The refined primary $\alpha-\mathrm{Mg}$ grains would benefit the feeding capability during solidification, reduce the possibility of shrinkage hole formation and improve their distribution, as shown in Fig.5c and 5d. In addition, the eutectic phase of $\mathrm{Mg}_{17} \mathrm{Al}_{12}$ was refined as well with high shearing, and its average size is decreased from $20 \mu \mathrm{m}$ to $10 \mu \mathrm{m}$, as shown in Fig. 5a and 5b. The refinement of primary $\alpha-\mathrm{Mg}$ and $\mathrm{Mg}_{17} \mathrm{Al}_{12}$ phase contributes the improvement of tensile strength and elongation, and the decreasing of shrinkage hole benefits the elongation improvement and variation reduction.

As a typical characteristic of HPDC, the formation of defect band occurs during solidification within die cavity. A formation mechanism of defect band had been proposed by A. K. Dahle and D.H. StJohn [5]. It was considered that the defect band was mainly consisted of coarse primary phases, segregation of solution elements and porosity. Therefore, the metallurgical quality of defect band with less porosity and segregation will have a positive effect on the mechanical properties of materials. 

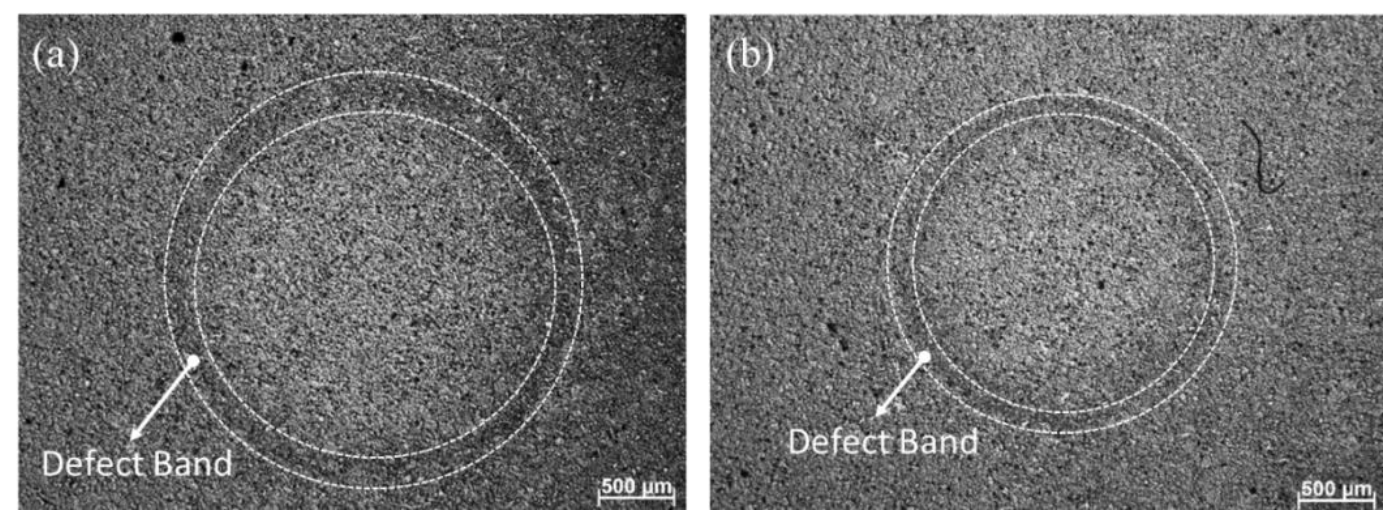

Figure 6 SEM micrographs showing the size and morphology of defect band formed in the cylinder tensile sample of the HPDC AZ91D alloy (a) without and (b) with high shearing being imposed.

The size and morphology of defect band of AZ91D alloy with HPDC and MC-HPDC are shown in Fig. $6 \mathrm{a}$ and $6 \mathrm{~b}$ respectively. It can be seen that the outer diameter of defect band is reduced from $2.7 \mathrm{~mm}$ to $2.0 \mathrm{~mm}$ for AZ91D alloy after application of high shearing. Correspondingly, the thickness of defect band is decreased from $300 \mu \mathrm{m}$ to $200 \mu \mathrm{m}$ after employing of high shearing technology. The formation of defect band is related to the value of solid fraction in melt, at a critical value, the crystals impinge on one another and would form a loose network of solid and then resistant to the melt flow. The solid fraction is a strong function of the size and shape of crystals, its value could vary from $15 \%$ for highly branched equiaxed dendrite to $50 \%$ for globular crystal [36]. In present study, the size of both coarse and fine primary $\alpha-\mathrm{Mg}$ grains of the HPDC AZ91D alloy is much bigger than that of alloy with MC-HPDC (as shown in Fig.3, Fig. $5 \mathrm{a}$ and $5 \mathrm{~b}$ ). This indicates that the defect band network of HPDC AZ91D alloy forms at lower solid fraction and at the earlier solidification stage in die cavity. In view of the solidification sequence of HPDC contour paralleled to the surface of the casting, the 
size of defect band with HPDC should be bigger than that of MC-HPDC, as shown in Fig.6a and $6 b$.

After completion of filling process during HPDC, the extreme high pressure of about 50 MPa-100 MPa was conducted and the solidification was occurred under such high pressure. With the high pressure, the feeding channels would be established from sample center to the previous solidification part, and the band would be refilled with liquid melt at this period. The liquid fraction in defect band would affect the subsequently solidification and determine the segregation and porosity. The higher liquid fraction would lead the band to be the last part of solidification than that in adjacent regions. Therefore, shrinkage porosity would form in the band without adequately liquid compensation. As an improving approach of high shear melt conditioning, the fine solid distribution in the band would reduce the formation possibility of porosity by forcing the band solidification prior to the adjacent regions.
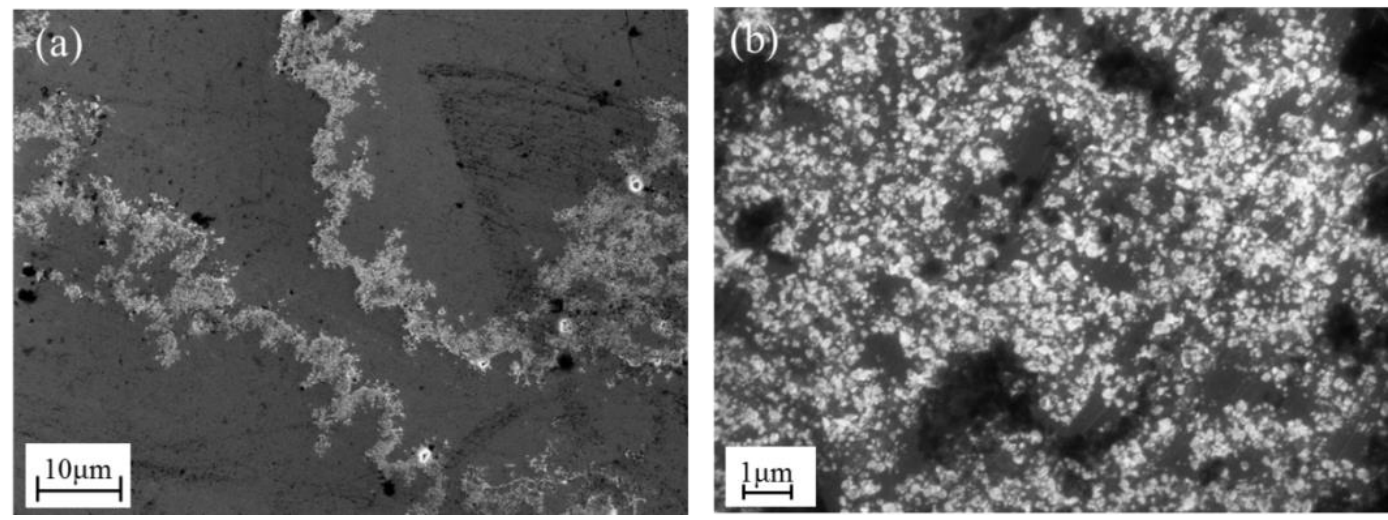

Figure 7 SEM micrographs showing the morphology of oxides in AZ91D melt (a) without and (b) with high shearing melt conditioning. 
The fundamental mechanism of high shearing on grain refinement is that it can disperse the $\mathrm{MgO}$ film to $\mathrm{MgO}$ particles efficiently by very high shear rate of about $10^{5} \mathrm{~s}^{-1}$ [37], and the dispersed $\mathrm{MgO}$ particles are the potency heterogeneous nuclei for $\alpha-\mathrm{Mg}$ grains during solidification [31]. During high shear processing, the melt was sucked into rotor-stator chamber from the bottom of high shear unit, and forced to enter the shear region to suffer the high shear rate and then pass through the drain holes to complete one cycle of high shearing. In view of very little amount of oxide film remained in $\mathrm{Mg}$ melt, it is very difficult to find and observe the oxides directly from the normal workpiece. Therefore, pressure filtration method was employed to concentrate the impurity in melt to facilitate analysis of its morphology, and then to assess the dispersive performance of high shearing. Fig.7a and $7 \mathrm{~b}$ show the morphology of oxide film and $\mathrm{MgO}$ particles for the AZ91D alloy without and with high shearing respectively. Without shearing, the long and coarse oxide films were found in the melt and consisted of agglomeration of fine $\mathrm{MgO}$ particles [28,32], the entrapment of such kind of film will deteriorate the properties and affect the quality of products. With proper high shear melt conditioning, the oxide film was breakdown and dispersed into individual particles with maximum size of 500nm, as shown in Fig. $7 \mathrm{~b}$. To harness the $\mathrm{MgO}$ particles as the heterogeneous nucleation sites for AZ91D alloy, it is necessary that there are an orientation relationship and small misfit between $\mathrm{MgO}$ and $\mathrm{Mg}$ matrix.

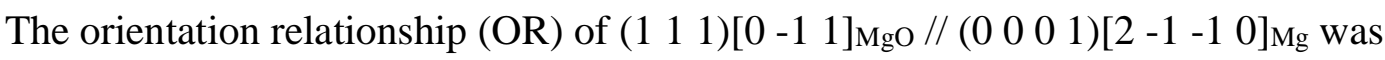
found (as shown in Fig.8) and, according to the atomic spacing which are experimentally measured to be $0.2991 \mathrm{~nm}$ for $\mathrm{MgO}$ and $0.3165 \mathrm{~nm}$ for $\alpha-\mathrm{Mg}$ in this work, the lattice misfit at the $\mathrm{MgO} / \mathrm{Mg}$ interface was therefore calculated to be $5.5 \%$ along the specific OR. This is in well agreement with the previous results reported by Wang et al. 
[32]. The potency of $\mathrm{MgO}$ particles on the grain refinement of pure $\mathrm{Mg}$ and AZ91D alloy was confirmed both from experimental and theoretical point of view $[28,29,31,32,35]$. According to the Free-growth model, the size of nuclei is inverse proportional to the supercooling [11]. Therefore, this kind of well-dispersed nano particles could nucleate $\alpha-\mathrm{Mg}$ with proper supercooling. HPDC process as a special casting method can supply a high speed filling and a solidification with high cooling rate of about $500-1000{ }^{\circ} \mathrm{C} / \mathrm{s}$. The large cooling rate could offer enough thermal supercooling to enable the nano $\mathrm{MgO}$ particles act as active nuclei.
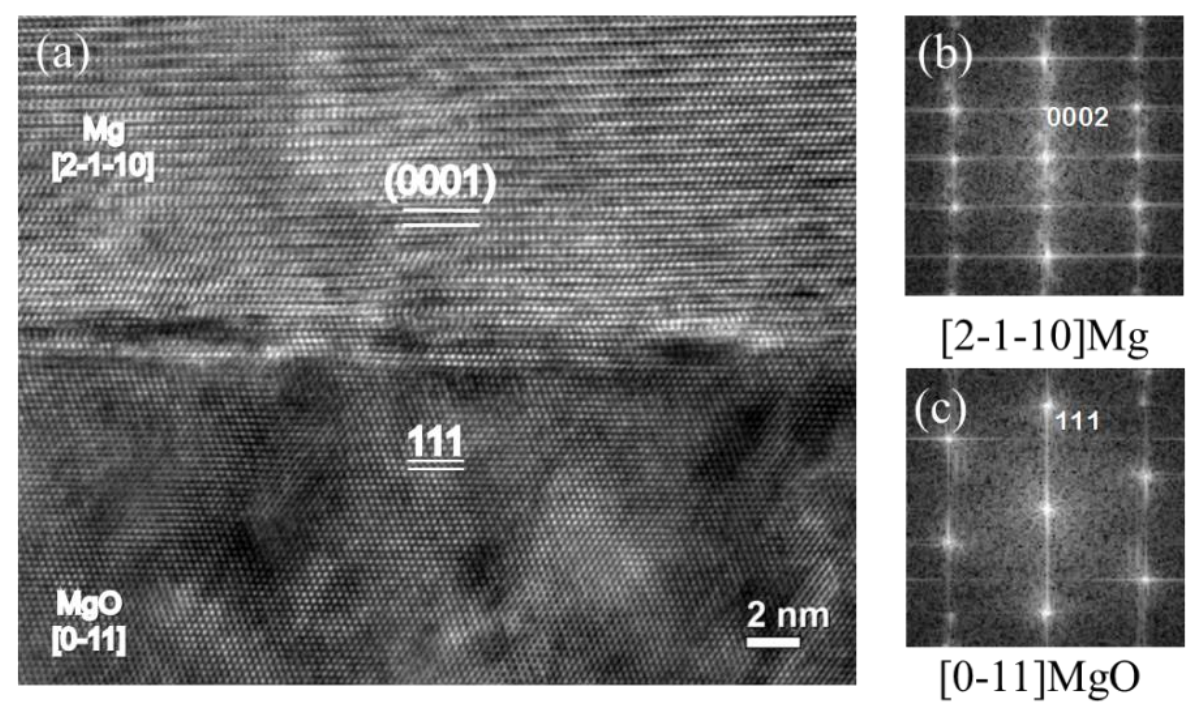

Figure 8 (a) HRTEM image showing the interface between $\mathrm{MgO}$ particle and $\alpha-\mathrm{Mg}$ matrix, (b) and (c) FFT patterns for $\mathrm{Mg}$ and $\mathrm{MgO}$ respectively.

The application of high shear melt conditioning for HPDC can refine the primary $\alpha-\mathrm{Mg}$ formed in both shot sleeve and die cavity via enhanced heterogeneous nucleation, and result in the refinement of the $\mathrm{Mg}_{17} \mathrm{Al}_{12}$ phase, reduction of the porosity and minimization of defect band. Therefore, the deterioration of mechanical properties was 
minimized, and the improvement of properties and the reduction of property variation could be achieved.

\section{Conclusions}

In summary, the melt conditioning treatment by high shearing can not only enhance the tensile strength and elongation simultaneously, but also improve the variation of yield strength and elongation of AZ91D alloy prepared by HPDC process. With MC-HPDC, the variation of elongation has a significant reduction from $31.2 \%$ for tradition HPDC to 19.7\%. Meanwhile, the variation of yield strength has a light improvement from $3.95 \%$ to $3.84 \%$. SEM observation indicates that the improvement of variation is attributed to the grain size reduction of primary $\alpha-\mathrm{Mg}$ formed in both shot sleeve and die cavity, the enhancement of metallurgical quality of defect band and refinement of $\mathrm{Mg}_{17} \mathrm{Al}_{12}$ eutectic phases caused by the grain refinement. SEM and HR-TEM observations reveal further that inoculation by the numerous well-dispersed $\mathrm{MgO}$ particles from breaking-up of the nature oxide films were the fundamental principle to the effective grain refinement and thus to the improvement of the variation.

\section{Acknowledgements}

This work was supported by EPSRC UK (Grant number EP/N007638), United Kingdom.

\section{Data availability}


The raw data required to reproduce these findings are available to download from [https://data.mendeley.com/datasets/w5tshrbxw4/draft?a=8dcb579c-a531-4998-b1bc-24 $62 \mathrm{dfc} 3 \mathrm{~d} 93 \mathrm{~d}]$.

\section{References}

[1] K.V. Yang, M.A. Easton, C.H. Cáceres, The development of the skin in HPDC Mg-Al alloys, Mater. Sci. Eng. A. 580 (2013) 191-195.

[2] X.Li, S.M. Xiong, Z. Guo, Improved mechanical properties in vacuum-assist high pressure die casting of AZ91D alloy, J. Mater. Process. Technol. 231 (2016) $1-7$.

[3] S. Otarawanna, H.I. Laukli, C.M. Gourlay, A.K. Dahle, Feeding mechanisms in high-pressure die castings, Metall. Mater. Trans. A. 41 (2010) 1836-1846.

[4] A.K. Dahle, S. Sannes, D.H. StJohn, H. Westengen, Formation of defect bands in high pressure die cast magnesium alloys, J. Light Met. 1 (2001) 99-103.

[5] A.K. Dahle, D.H. StJohn, Rheological behaviour of the mushy zone and its effect on the formation of casting defects during solidification, Acta Mater. 47 (1999) $31-41$.

[6] X. Li, S.M. Xiong, Z. Guo, On the tensile failure induced by defect band in high pressure die casting of AM60B magnesium alloy, Mater. Sci. Eng. A. 674 (2016) $687-695$.

[7] W. Ha, Y.J. Kim, Effects of cover gases on melt protection of Mg alloys, J. Alloys Compd. 422 (2006) 208-213. 
[8] A.L. Greer, P.S. Cooper, M.W. Meredith, W. Schneider, P. Schumacher, J.A. Spittle, A. Tronche, Grain Refinement of Aluminium Alloys by Inoculation, Adv. Eng. Mater. 5 (2003) 81-91.

[9] C. Limmaneevichitr, W. Eidhed, Fading mechanism of grain refinement of aluminum-silicon alloy with Al-Ti-B grain refiners, Mater. Sci. Eng. A. 349 (2003) 197-206.

[10] M. Easton, David StJohn, Grain Refinement of Aluminum Alloys Part I.The nucleant and solute paradigms-A review of the literature, Met. Mater. Trans. A. 31A (1999) 1613-1623.

[11] A.L. Greer, A.M. Bunn, A. Tronche, P. V. Evans, D.J. Bristow, Modelling of inoculation of metallic melts: application to grain refinement of aluminium by Al-Ti-B, Acta Mater. 48 (2000) 2823-2835.

[12] P.S. Mohanty, J.E. Gruzleski, Grain refinement mechanisms of hypoeutectic Al-Si alloys, Acta Mater. 44 (1996) 3749-3760.

[13] P.S. Mohanty, J.E. Gruzleski, Mechanism of grain refinement in aluminium, Acta Metall. Mater. 43 (1995) 2001-2012.

[14] M. Qian, A. Das, Grain refinement of magnesium alloys by zirconium : Formation of equiaxed grains, Scr. Mater. 54 (2006) 881-886.

[15] R. Gunther, C. Hartig, R. Bormann, Grain refinement of AZ31 by (SiC)P: Theoretical calculation and experiment, Acta Mater. 54 (2006) 5591-5597. 
[16] B. Nagasivamuni, K.R. Ravi, An analytical approach to elucidate the mechanism of grain refinement in calcium added Mg-Al alloys, J. Alloys Compd. 622 (2015) 789-795.

[17] Y. Zeng, B. Jiang, D. Huang, J. Dai, F. Pan, Effect of Ca addition on grain refinement of Mg-9Li-1Al alloy, J. Magnes. Alloy. 1 (2013) 297-302.

[18] M. Yang, F. Pan, R. Cheng, A. Tang, Effect of Mg-10Sr master alloy on grain refinement of AZ31 magnesium alloy, Mater. Sci. Eng. A. 491 (2008) 440-445.

[19] M. Suresh, A. Srinivasan, K.R. Ravi, U.T.S. Pillai, B.C. Pai, Influence of boron addition on the grain refinement and mechanical properties of AZ91 Mg alloy, Mater. Sci. Eng. A. 525 (2009) 207-210.

[20] S. Liu, Y. Chen, H. Han, Grain refinement of AZ91D magnesium alloy by a new Mg-50\% Al4 $\mathrm{C}_{3}$ master alloy, J. Alloys Compd. 624 (2015) 266-269.

[21] M. Suresh, A. Srinivasan, U.T.S. Pillai, B.C. Pai, Mechanism for grain refinement and mechanical properties of AZ91 Mg alloy by carbon inoculation, Procedia Eng. 55 (2013) 93-97.

[22] M. Suresh, A. Srinivasan, K.R. Ravi, U.T.S. Pillai, B.C. Pai, Microstructural refinement and tensile properties enhancement of $\mathrm{Mg}-3 \mathrm{Al}$ alloy using charcoal additions, Mater. Sci. Eng. A. 528 (2011) 2502-2508.

[23] Y.M. Kim, L. Wang, B.S. You, Grain refinement of Mg-Al cast alloy by the addition of manganese carbonate, J. Alloys Compd. 490 (2010) 695-699. 
[24] L. Lu, A.K. Dahle, D.H. Stjohn, Grain refinement efficiency and mechanism of aluminium carbide in Mg-Al alloys, Scr. Mater. 53 (2005) 517-522.

[25] H. Ding, H. Li, X. Liu, Different elements-induced destabilisation of TiC and its application on the grain refinement of $\mathrm{Mg}$-Al alloys, J. Alloys Compd. 485 (2009) 285-289.

[26] Q. Zhang, B. Liu, Z. Niu, Z. Zhang, Z. Leng, Grain refinement and mechanical properties of Mg-5Li-3Al alloy inoculated by Al-5Ti-1B master alloy, Mater. Sci. Eng. A. 619 (2014) 152-157.

[27] S. Liu, Y. Zhang, H. Han, B. Li, Effect of Mg-TiB 2 master alloy on the grain refinement of AZ91D magnesium alloy, J. Alloys Compd. 487 (2009) 202-205.

[28] Z. Fan, Y. Wang, M. Xia, S. Arumuganathar, Enhanced heterogeneous nucleation in AZ91D alloy by intensive melt shearing, Acta Mater. 57 (2009) $4891-4901$.

[29] S. Tzamtzis, H. Zhang, N. Hari Babu, Z. Fan, Microstructural refinement of AZ91D die-cast alloy by intensive shearing, Mater. Sci. Eng. A. 527 (2010) $2929-2934$.

[30] S. Tzamtzis, H. Zhang, M. Xia, N.H. Babu, Z. Fan, Recycling of high grade die casting AM series magnesium scrap with the melt conditioned high pressure die casting (MC-HPDC) process, Mater. Sci. Eng. A. 528 (2011) 2664-2669.

[31] H. Men, B. Jiang, Z. Fan, Mechanisms of grain refinement by intensive shearing of AZ91 alloy melt, Acta Mater. 58 (2010) 6526-6534. 
[32] Y. Wang, Z. Fan, X. Zhou, G.E. Thompson, Characterisation of magnesium oxide and its interface with $\alpha-\mathrm{Mg}$ in $\mathrm{Mg}-\mathrm{Al}$-based alloys, Philos. Mag. Lett. 91 (2011) 516-529.

[33] S. Ji, W. Yang, B. Jiang, J.B. Patel, Z. Fan, Weibull statistical analysis of the effect of melt conditioning on the mechanical properties of AM60 alloy, Mater. Sci. Eng. A. 566 (2013) 119-125.

[34] The aluminum Association, Standard test procedure for aluminum alloy grain refiners, (1990).

[35] M. Xia, S. Mitra, D. Brij, G. Liu, Z. Fan, Melt-conditioned, high-pressure die casting of Mg-Zn-Y Alloy, Metall. Mater. Trans. B. 41 (2010) 209-213.

[36] C.M. Gourlay, A.K. Dahle, Dilatant shear bands in solidifying metals, Nature. 445 (2007) 70-73.

[37] M. Tong, J.B. Patel, I. Stone, Z. Fan, D.J. Browne, Identification of key liquid metal flow features in the physical conditioning of molten aluminium alloy with high shear processing, Comput. Mater. Sci. 131 (2017) 35-43. 
Figure 1 Grain refinement assessed by TP-1 method showing the trend of grain size of AZ91D alloy with various shearing time.

Figure 2 Tensile test of HPDC samples showing the distribution of elongation and yield strength of AZ91D alloys with and without high shearing application.

Figure 3 Microstructure of AZ91D HPDC tensile samples showing the coarse primary $\alpha-M g$ grains formed in shot sleeve (a) with and (b) without high shear melt conditioning, and (c) the grain size distribution of coarse primary $\alpha-\mathrm{Mg}$ formed in shot sleeve with and without high shearing.

Figure 4 Microstructures showing the grain size of AZ91D ingot solidified inside shot sleeve (a) without and (b) with high shearing application.

Figure 5 SEM micrographs showing the microstructure of $\alpha$-Mg grains solidified inside the die cavity and the porosity distribution of the AZ91D alloy (a, c) without and (b, d) with high shearing being imposed.

Figure 6 SEM micrographs showing the size and morphology of defect band formed in the cylinder tensile sample of the HPDC AZ91D alloy (a) without and (b) with high shearing being imposed. 
Figure 7 SEM micrographs showing the morphology of oxides in AZ91D melt (a) without and (b) with high shearing melt conditioning.

Figure 8 (a) HRTEM image showing the interface between $\mathrm{MgO}$ particle and $\alpha-\mathrm{Mg}$ matrix, (b) and (c) FFT patterns for $\mathrm{Mg}$ and $\mathrm{MgO}$ respectively.

Table 1 The average value, standard deviation and coefficient of variation of yield strength and elongation of AZ91D alloy with HPDC and MC-HPDC. 